\title{
Circuit
}

Musiques contemporaines

\section{Portrait de Réseaux des arts médiatiques}

\section{Réjean Beaucage}

Volume 16, numéro 3, 2006

À musique contemporaine, supports contemporains?

URI : https://id.erudit.org/iderudit/902417ar

DOI : https://doi.org/10.7202/902417ar

Aller au sommaire du numéro

Éditeur(s)

Les Presses de l'Université de Montréal

ISSN

1183-1693 (imprimé)

1488-9692 (numérique)

Découvrir la revue

Citer cet article

Beaucage, R. (2006). Portrait de Réseaux des arts médiatiques. Circuit, 16(3), 106-116. https://doi.org/10.7202/902417ar d'utilisation que vous pouvez consulter en ligne.

https://apropos.erudit.org/fr/usagers/politique-dutilisation/ 


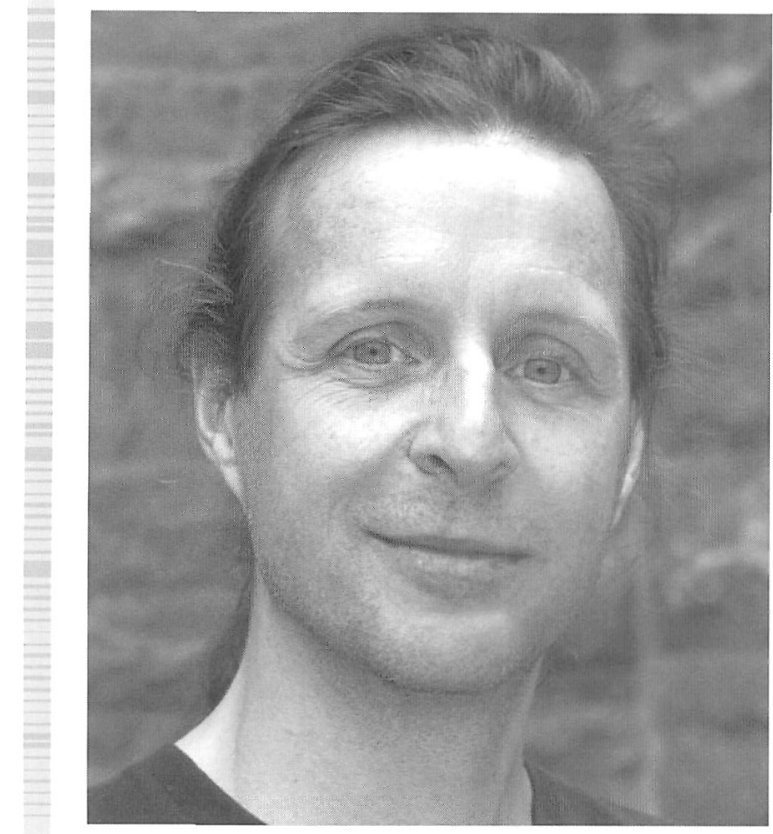

Jean-François Denis

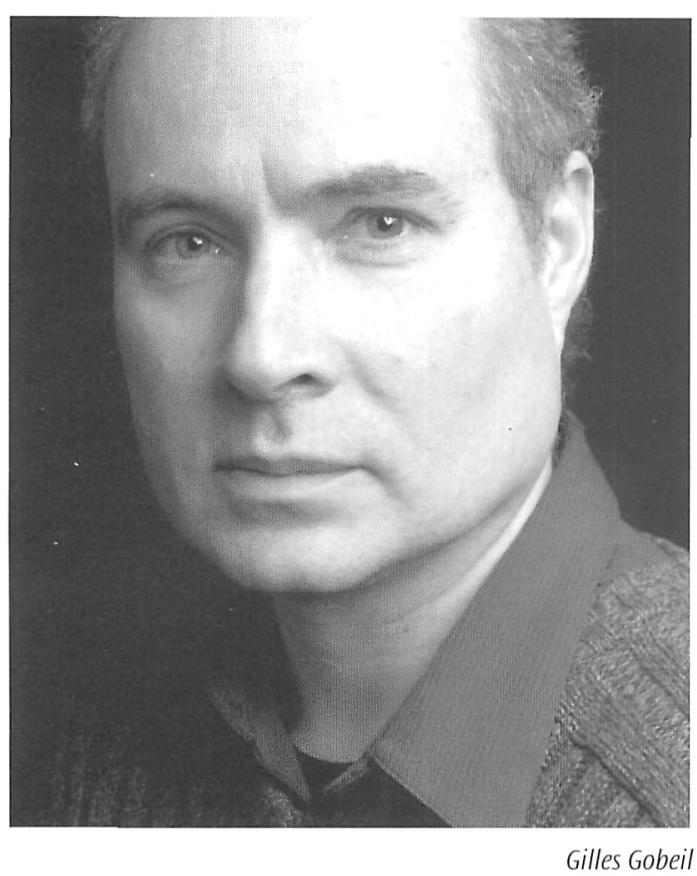

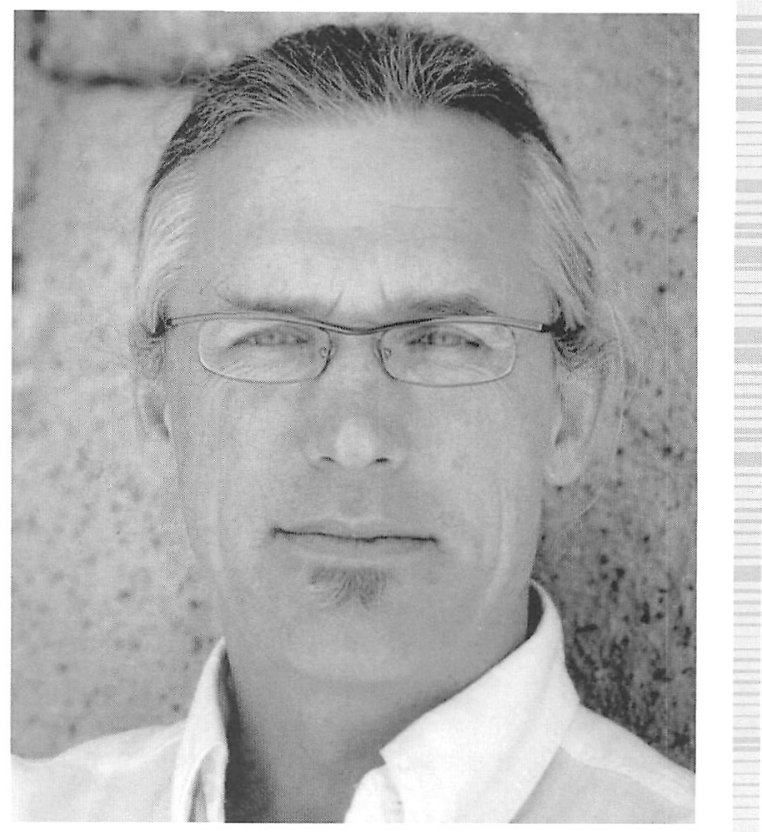

Robert Normandeau

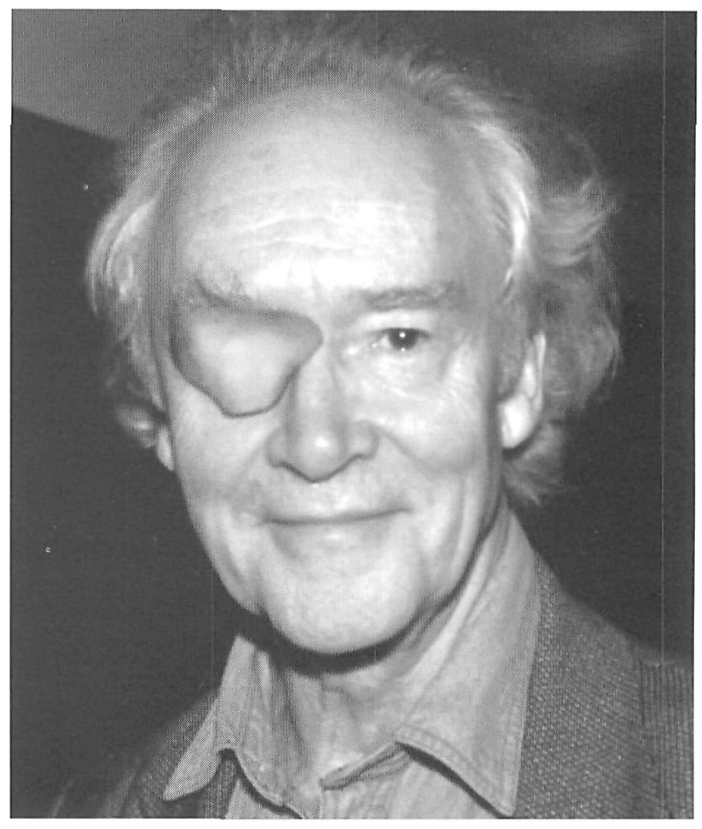

Francis Dhomont

106 


\section{Portrait de Réseaux des arts médiatiques}

par Réjean Beaucage

Quinze ans d'existence, pour une société de concerts, ce n'est pas mal. Et quand elle fait la promotion d'une musique réputée difficile, c'est même très bien. Rétrospective en forme de coup de chapeau pour la société de concerts Réseaux, qui passe ce cap cette année.

\section{* *}

Si la musique concrète naît à Paris en 1948 et que fleurissent ensuite un peu partout en Europe des studios de musique électroacoustique, ce n'est qu'en 1964 qu'apparaît à Montréal, et au Québec, le premier studio institutionnel de ce genre'. II se nomme Electronic Music Studio (EMS) et est installé à l'Université McGill grâce aux démarches effectuées par le compositeur István Anhalt. Suivront ensuite l'Université Laval à Québec, avec le premier studio francophone (1969), puis l'Université Concordia (1971), l'Université de Montréal, qui équipe à partir de 1974 le secteur électroacoustique de sa faculté de musique et, enfin, les Conservatoires de musique de Québec (1978) et de Montréal (1979)². On pouvait composer de la musique électroacoustique dans ces studios, ou apprendre à le faire. Mais il fallait aussi pouvoir la faire entendre au public en dehors des concerts donnés dans les institutions d'enseignement.

C'est la raison qui poussa en 1978 Yves Daoust, Marcelle Deschênes, Philippe Ménard, Michel Longtin et Pierre Trochu à fonder un regroupement de compositeurs dont le but êtait de favoriser le développement de la musique électroacoustique aux côtés des autres formes de musique contemporaine. Ainsi naquit l'Association pour la recherche et la création électroacoustiques du Québec (ACREQ), premier organisme canadien entièrement consacré au développement de cette musique. D'autres organismes appuieront bientôt l'ACREQ dans sa démarche, comme Les Événements du Neuf (1978 aussi), où sont présentés à l'occasion des concerts électroacoustiques entre les concerts instrumentaux, ou le Group of the Electronic Music Studio (GEMS), fondé à McGill par alcides lanza, John Oliver et Claude Schryer en 1983. Dans les différents organismes comme à l'ACREQ, la programmation se diversifiera au fil du temps. Le terme électroacoustique ne servira bientôt plus exclusivement à désigner la diffusion de pièces 
sur bande, comme ce pouvait être le cas auparavant, mais en viendra de plus en plus à désigner les différentes formes de musiques mixtes (instrumentale avec bande ou traitements en temps réel). Bref, les programmations de concert se diversifient afin de rendre compte de la multiplicité des nouvelles approches compositionnelles qu'offrent les développements techniques et cela se fait au détriment de la musique sur bande ${ }^{3}$. En 1993, le compositeur Alain Thibault prend la direction artistique de l'ACREQ à la suite de Claude Schryer et fait connaître son intention de " ramener les humains sur la scène". II le fera avec beaucoup de succès en produisant cette année-là l'ensemble Dangerous Kitchen, qui réunit 10 instrumentistes sous la direction de Walter Boudreau pour interpréter des œuvres du compositeur américain Frank Zappa.

Au début des années 1990, trois compositeurs intéressés principalement par la musique sur support et, plus précisément, par la musique acousmatique ${ }^{4}$, choisissent de fonder un organisme voué exclusivement à ce type de musique. Ce sont Jean-François Denis, Gilles Gobeil et Robert Normandeau, et l'organisme en question prend le nom de Réseaux des arts médiatiques (mieux connu sous l'abréviation Réseaux). Ils décident d'inaugurer l'organisme le 2 novembre 1991 en saluant un compositeur qui en a inspiré bien d'autres par son travail et son enseignement, mais dont les œuvres restent difficiles à trouver. Difficiles à trouver? Pas pour longtemps! Jean-François Denis a produit le premier disque de l'étiquette qu'il a fondée avec le compositeur Claude Schryer, Empreintes DIGITALes, en janvier 1990. Le concert inaugural sera donc un concert-lancement.
Le concert est intitulé "Top secret" et le compositeur Francis Dhomont est invité à y diffuser une de ses pièces. Lorsqu'il se présente à la soirée, c'est pour s'apercevoir que tout le concert lui est consacré et que le disque lancé est un album double accompagné d'un magnifique livret rempli de témoignages de ses amis qui le saluent pour son $65^{\mathrm{e}}$ anniversaire! Personne n'aura vendu la mèche et ce concert-lancement reste sans doute aujourd'hui dans les annales des surprisesparties les plus réussies! Le double cD paru à cette occasion ${ }^{5}$ est aujourd'hui épuisé, mais chacun des disques a été réédité séparément par Empreintes DIGITALes.

En 1992, à l'occasion du $350^{\mathrm{e}}$ anniversaire de Montréal, Réseaux présente, entre le 19 mai et le 7 octobre, neuf soirées de concert au Planétarium de Montréal, un lieu que Robert Normandeau connaît bien puisqu'il y organise régulièrement depuis 1986 la série de concerts Clair de terre, produite par l'ACREQ. À cette occasion, il présente son œuvre Tangram, un titre qui sera celui de son premier disque, à paraître chez Empreintes DIGITALes, en 1994. Toujours en mai 1992, le 29, Réseaux participe à l'inauguration de la $5^{\mathrm{e}}$ salle de la Place des arts avec une autre prestation de Robert Normandeau qui diffuse pour l'occasion en création la pièce Fragments, une œuvre qui n'a pas à ce jour trouvé le chemin du disque.

C'est en février 1996 que Réseaux réalise une première collaboration internationale en participant à l'événement Québec-Belgique organisé par Codes d'accès (autrefois la Société des concerts alternatifs du Québec, ou SCAQ) et l'organisme belge Musiques \& Recherches. L'événement s'étend sur plusieurs jours et 


\section{Compositeurs ayant participé aux soirées acousmatiques de l'événement Québec-Belgique :}

Elizabeth Anderson (BE), Ingrid Drese (BE), Stephan Dunkelman (BE), Luc Fortin (QC), Gilles Gobeil $(\underline{Q C})$, Slavec Kwi (BE), Robert Normandeau $(\mathrm{QC})$, Jean-Louis Poliart (BE), Stéphane Roy (QC), Pierre Sainte-Marie $(\mathrm{QC})$, Jacques Tremblay $(\mathrm{QC})$, Marc Tremblay $(\mathrm{QC})$, Todor Todorof $(\mathrm{BE})$, Marie-Jeanne Wyckmans (BE).

deux soirées sont consacrées à la diffusion de musique acousmatique. On y découvre avec plaisir un panorama de compositeurs belges et québécois connus et moins connus. Les réseaux se construisent...

Une deuxième collaboration du même type sera établie en 2002 lors de l'événement Voyages : DublinMontréal, qui présentait bien entendu un programme d'œuvres irlandaises et québécoises. Il s'agissait d'une collaboration avec l'organisme Concerts $M$.

C'est en 1997 que débute l'aventure Rien à voir. L'expression populaire choisie comme titre à la série de concerts ne pourrait être plus exacte : en effet, au concert acousmatique, on laisse le minimum d'éclairage afin de permettre à l'auditeur de se placer en situation d'uécoute extrême" et... il n'y a rien à voir ${ }^{6}$. C'est cette série de concerts qui mettra vraiment Réseaux sur la carte des organismes qui comptent en musique contemporaine à Montréal. Organisés au rythme de deux par année, les concerts Rien à voir permettent un accès direct à des compositeurs qu'aucun autre organisateur ne songerait malheureusement à présenter au public montréalais. On pourrait sans doute, avec un peu de chance, assister de temps à autre à des concerts des compositeurs d'ici, mais Réseaux a mis l'accent sur ceux qui viennent d'ailleurs, non pas par soumission à une quelconque forme de colonialisme culturel, mais plutôt dans un but pédagogique. L'histoire de l'électroacoustique n'étant pas encore très longue (on fêtait en 1998 son cinquantenaire), Réseaux a pu présenter parmi ses invités des chercheurs qui furent de véritables pionniers de l'histoire de la musique et à qui le $x^{\mathrm{e}}$ siècle doit beaucoup. On pense à la branche française, bien sûr, avec les François Bayle, Michel Chion, Luc Ferrari, Bernard Parmegiani ou Jean-Claude Risset. Mais il y a aussi tous ceux que les moins fervents d'entre nous auront découvert là, les Jon Appleton (Américain), Beatriz Ferreyra (Argentine), Ảke Parmerud (Suèdois), Jonty Harrison (Anglais) ou Annette Vande Gorne (Belge), etc. Ces compositeurs sont presque toujours invités à participer à diverses activités universitaires (conférences, classes de maître) et présentent également des ateliers de diffusion?

\section{Compositeurs participants aux soirées acousmatiques de l'événement Voyages : Dublin-Montréal :}

John Bassetti (IR), Conor J Curran (IR), Jean-François Denis (QC), Donnacha Dennehy (IR), Roger Doyle (IR), Gilles Gobeil (QC), Robert Normandeau (QC), Stéphane Roy (QC). 


\section{Compositeurs ayant participé à la série de concerts Rien à voir :}

Randall Smith (CA) - Jonty Harrison (RU) - Yves Daoust (QC) - Michel Chion (FR)

Ned Bouhalassa (QC) - Åke Parmerud (SE) - Jon Appleton (EU) - Luc Ferrari (FR)

Mark Wingate (EU) - Christian Calon (QC) - Annette Vande Gorne (B) - Bernard Parmegiani (FR)

Stéphane Roy (QC) - Erik Michael Karlsson (SE) - Ludger Brümmer (ALL) - François Bayle (FR)

Monique Jean (QC) - Christian Zanési (FR) - Paul Dolden (QC) - Francis Dhomont (FR/QC)

Marc Tremblay (QC) - Jens Hedman et Paulina Sundin (SE) - Robert Normandeau (QC) - Denis Smalley (RU)

Louis Dufort (QC) - John Oswald (CA) - Natasha Barrett (Norv/RU) - Jean-Claude Risset (FR)

Chantal Laplante $(\underline{Q C})$

Adrian Moore (RU) - Régis Renouard Larivière (FR) - Gilles Gobeil ( $\mathrm{QC})$ - Beatriz Ferreyra (AR/FR)

Jacques Tremblay (QC) - Hans Tutschku (ALL) - John Young (NZ) - Francis Dhomont (FR/QC)

Ingrid Drese (BE) + Stephan Dunkelman (BE) - Jonty Harrison (RU) - Bernard Parmegiani (FR)

Wayne Siegel (EU/DK) - Flô Menezes (BR) - Elainie Lillios (EU) - Robert Normandeau (QC)

François Donato (FR) - Alistair MacDonald (ÉCOSSE) - Stéphane Roy (QC) - Åke Parmerud (SE)

Gilles Gobeil (QC) et René Lussier (QC) - Luigi Ceccarelli (IT) - Bernard Fort (FR) -

Ricardo Dal Farra (AR)

Randall Smith (CA) - Hildegard Westerkamp (CA) - Andrew Lewis (RU) - Yves Daoust (QC).

Réseaux n'a laissé sortir qu'une seule fois le concept de soirées Rien à voir hors de sa programmation régulière. Ce fut à l'occasion d'une autre collaboration avec un organisme de Montréal, Les Productions Supermémél Supermusique, durant un événement qui s'étalait sur trois semaines en octobre et novembre 2000. Hommage à la création des femmes au $x x^{e}$ siècle, l'événement SuperMicMac (pour Musiciennes Innovatrices Canadiennes - Musiques Actuelle et Contemporaine) consacrait deux soirées à la diffusion d'œuvres acousmatiques de compositrices canadiennes. Cette série de concerts Rien à voir (8) nous a permis d'entendre des œuvres de pionnières de l'électroacoustique au Québec comme Micheline Coulombe Saint-Marcoux ou Marcelle Deschênes, mais aussi des œuvres de jeunes compositrices, choisies par Chantal Laplante, programmatrice invitée. L'événement SuperMicMac a reçu le prix Opus du Conseil québécois de la musique dans la catégorie Événement musical de l'année.

Dès le début de la série des Rien à voir, Denis, Gobeil et Normandeau se sont trouvés devant un sérieux problème de logistique. Une telle série de concerts, tenue en des lieux peu adaptés (le Théâtre La Chapelle, pour les cinq premières éditions) requérait une solide équipe 


\section{Compositeurs ayant participé aux concerts Jeunes compositeurs ou Découverte :}

Nicolas Basque, Olivier Bélanger, Christian Bouchard, Nicolas Borycki, Nicolas Boucher, Guillaume Coutu Dumont, Jean-François Dessureault, Jean-Sébastien Durocher, Stephen De Oliveira, Louis Dufort, Georges Forget, Alain Gauthier, Yves Gigon, François Girouard, Andrea Holtslander, Mathieu Lafontaine, Justine Lamoureux, Martin Leclerc, Mathieu Marcoux, Martin Marier, Alexandre Menachi, Nicolas Orton, Sylvain Pohu, Jean-Michel Robert, Éric Rocheleau, Julien Roy, Pierre Alexandre Tremblay, David Turgeon, Paul W Williams.

technique afin d'être menée à bien ${ }^{8}$ et ils décidèrent d'embaucher des étudiants en électroacoustique pour le faire. Ces derniers sont bien entendu payés pour leurs services, passent leurs journées en compagnie de grands compositeurs dont certains font de la diffusion depuis une quarantaine d'années et, en prime, on leur offre un concert afin que chacun d'eux puisse diffuser l'une de ses pièces. Les techniciens qui firent un succès de la première édition de Rien à voir (Pierre Alexandre Tremblay, Nicolas Boucher, Éric Rocheleau, Alain Gauthier, Louis Dufort) ont tous poursuivi la carrière de compositeur'9 Depuis la dixième édition de Rien à voir, les fondateurs de Réseaux ont voulu prendre en compte l'évolu- tion parallèle d'une musique techno qui est souvent le fruit de compositeurs autodidactes qui se réclament de I'héritage de Pierre Schaeffer et consorts. II y eut des concerts Post-rien et Pré-rien, chaque fois à la Casa del Popolo, située boulevard Saint-Laurent à Montréal, en face de l'Espace Go, où étaient donnés les Rien à voir. Ce rapprochement indique bien l'antisectarisme de l'organisme et son ouverture face aux nouvelles façons de concevoir la musique électroacoustique.

Bien que les trois fondateurs de Réseaux aient choisi de se donner le mandat spécifique de "se consacrer principalement à l'art acousmatique, genre musical sur support (bande magnétique, disquette, etc.) présenté

\section{Compositeurs et collectifs ayant participé aux concerts Post-rien et Pré-rien :}

Aimé Dontigny, Alexander MacSween, Alexandre St-Onge, Alphacat (EU), Books on Tape (EU), Cal Crawford, Camp, Chris Degiere, Click Tracy (EU), Coin Gutter, David Turgeon, David Turgeon + Shane Turner, Dick Richards, Diskette (EU), Dustin Craig, Érick Dorion, Eryk Salvaggio, Estevan Benson, Félix Boisvert, François Handfield, Guillaume Théroux, I8U et Félix Frédéric Baril, James Schidlowsky, Jean-Michel Robert, Jeff Morton, John Phillips, Jon Vaughn, Jon Vaughn + Nicolas Bernier, Julie Rousse, Kevin M Krebs, Kevin M Krebs + Aimé Dontigny, Komsomolsk, L'orchestre de granulation, Louis Dufort, Theodoros Lotis, Louis Trottier, Magali Babin, Guillaume Rancourt, Martin Leclerc, Mathieu Marcoux, Max Haiven, Michel F. Côté, Noah Sasso, CEuf Korreckt, Olivier Bélanger, Philémon Girouard, Ryan Patterson, Sam Shalabi, Sam Shalabi + Martin Bédard, Samiland, The Abstrakters, themoonstealingproject (EU), Todd Drootin, While (EU). 


\section{Compositeurs ayant participé aux concerts Parr(A)cousmatique :}

Arturo Parra (QC) et Mauricio Bejarano (CO), Francis Dhomont (FR/QC), Gilles Gobeil (QC), Robert Normandeau $(\mathrm{QC})$, Stéphane Roy $(\mathrm{QC})$.

sur un orchestre de haut-parleurs ${ }^{10}$ ", ils ne sont pas dogmatiques au point de s'interdire toute incursion dans des domaines musicaux connexes ${ }^{11}$. Ainsi, en mai 1998, Réseaux présente un concert de musique mixte au Festival International de Musique Actuelle de Victoriaville (FIMAV). Le guitariste d'origine colombienne Arturo Parra est sur la scène et interagit avec un enregistrement. Chaque pièce a été composée pour que la partie sur support puisse se suffire à elle-même, mais lors du concert le guitariste y ajoute bien entendu sa touche personnelle. Le concert sera repris en novembre au Théâtre La Chapelle, à Montréal, avec une pièce supplémentaire offerte par un compatriote de Parra, Mauricio Bejarano ${ }^{12}$.

Réseaux continuera à étendre ses contacts en produisant en 1999 l'exact contraire de Rien à voir, soit Plein la $v u e$, un concert de musique mixte qui donnera en effet à voir le guitariste René Lussier, dans un work in progress composée avec Gilles Gobeil : Le contrat. Ce concert offrira également une très rare occasion d'entendre une œuvre du regretté compositeur français Luc Ferrari, soit l'obsédante Cellule 75 Force du rythme et cadence forcée, interprétée par le pianiste Jacques Drouin et le percussionniste Julien Grégoire. Drouin interprétera aussi les Figures de rhétorique de Robert Normandeau.
La musique mixte, on en convient, en raison des musiciens sur scène, n'est pas acousmatique. Elle offre néanmoins au compositeur de musique électroacoustique - à condition de pas être monomaniaque — des plaisirs certains. C'est pourquoi Réseaux poursuivra dans cette optique en 2001 avec un concert intitulé... Flûte! un concert... Sur scène, cette fois, apparaît la flûtiste Claire Marchand qui accompagne la bande (ou est-ce le contraire?).

Cette approche culminera en février 2002 par une association avec le Nouvel Ensemble Moderne pour lancer MusMix, la Tribune canadienne de musiques mixtes, événement qui se veut biennal et qui permet aux deux organismes de présenter un répertoire malheureusement trop négligé, celui pour ensemble et bande, et ce, dans des conditions optimums.

En 2001, Robert Normandeau rédige à la demande du Conseil des Arts du Canada une étude intitulée Situation de l'électroacoustique au Canada; bien qu'il puisse sembler réjouissant à un habitant de Montréal, ville historiquement très active en matière de musique électroacoustique, le portrait n'est pas rose et Normandeau démontre, par exemple, que l'aide financière accordée au développement de ce secteur est dérisoire en comparaison des budgets disponibles en

\section{Compositeurs ayant participé au concert Flûte ! un concert... :}

Christian Calon, Louis Dufort, Monique Jean, Shirish Korde, Robert Normandeau, Guy Pelletier. 


\section{Compositeurs ayant participé aux concerts MusMix 1 et 2 :}

Serge Arcuri, Martin Bédard, Peter Hannan, Monique Jean, Jean-François Laporte, alcides lanza, Farangis Nurulla-Khoja, John Oliver, Laurie Radford, Andrew Staniland, Kotoka Suzuki.

musique (en 1999-2000, le Conseil des arts et des lettres du Québec [CALQ] consacre 0,3 de 1\% de son budget "musique" aux deux organismes voués au développement de la musique électroacoustique au Québec l'ACREQ et Réseaux - tandis que l'auteur estime la part du Conseil des Arts du Canada [CAC] dévolue à l'électroacoustique à 0,7 de $1 \%$ de son budget consacré à la musique).

Quelques jours avant de présenter Rien à voir (13), soit les 2, 3 et 8 mars 2003, Réseaux produisait dans le cadre du Festival international Montréal/Nouvelles Musiques (MNM) un concert de musique mixte (du compositeur Yves Daoust), un concert acousmatique (avec les compositeurs Ned Bouhalassa, Francis Dhomont, Alain Gauthier, Monique Jean et Marc Tremblay) et coproduisait le concert de la $15^{\mathrm{e}}$ édition du Concours national des jeunes compositeurs de Radio-Canada (où furent entendues des œuvres de Félix Boisvert, Jean-Michel Robert, Jacques Tremblay et Louis Trottier). Réseaux participait aussi à la deuxième édition de MNM en 2005 en invitant les compositeurs français François Bayle et Bernard Parmegiani et en offrant à Gilles Gobeil la possibilité de faire entendre ses œuvres mixtes avec ondes Martenot (Suzanne Binet-Audet, ondiste). C'est pendant MNM, le 3 mars 2003, que Réseaux lançait les iConcerts, une formule utilisant les ressources de l'Internet pour rendre disponibles par téléchargement les 17 œuvres commandées à autant de compositeurs depuis la fondation de la société. Les œuvres seront programmées dans six concerts imaginaires, dévoilés à un rythme mensuel (les œuvres sont toujours en ligne sur le site de Réseaux : $<$ www.rien.qc.ca>).

Après la $15^{e}$ édition de Rien à voir, les animateurs de Réseaux ont senti le besoin de renouveler la formule... par un retour aux sources, puisque la série Pulsar, présentée deux fois - d'abord avec des œuvres de Robert Normandeau, puis du français Jean-Claude Éloy - nous ramenait au Planétarium de Montréal.

Essoufflement du public? Effritement de la pertinence de la proposition acousmatique? Les temps sont petit à petit devenus plus difficiles pour le type de concerts que présentait Réseaux, et les animateurs de la société de concerts ont imaginé une autre série de concerts : Akousma, qui, contrairement à ce que son

\section{Compositeurs dont la musique peut-être entendue sur le site iConcerts :}

Nicolas Borycki, Nicolas Boucher, Ned Bouhalassa, Christian Calon, Francis Dhomont, Paul Dolden, Alain Gauthier, Monique Jean, Elainie Lillios, Robert Normandeau, John Oswald, Éric Rocheleau, Stéphane Roy, Wayne Siegel, Randall Smith, Marc Tremblay, Pierre Alexandre Tremblay. 


\section{Artistes invités à se produire dans le cadre de la série Akousma :}

Patrick Ascione (FR) - Ned Bouhalassa (QC) - Marcelle Deschênes (QC) - Louis Dufort (QC) - John Oliver (CA) - Pierre Alexandre Tremblay (QC) - Annette Vande Gorne (BE) - Theresa Transistor (QC) - soirées danse-musique avec Lucie Grégoire $(\mathrm{QC})$ et Robert Normandeau $(\mathrm{QC})$ - installation sonore de Roxanne Turcotte $(\mathrm{QC})$.

titre semble indiquer, ne présente pas que de la musique acousmatique, mais tout l'éventail électroacoustique, de l'installation au spectacle danse-musique et de la musique mixte à la vidéomusique, en passant, bien entendu, par l'acousmatique. La première édition d'Akousma s'est tenue en janvier 2005 et la deuxième, en décembre de la même année.

En juin 2006, Réseaux, après avoir souvent plongé son public dans la pénombre acousmatique, le plongeait carrément dans l'eau pour les concerts sub $\bullet a \bullet q u a t \bullet i c$, présentés par le duo montréalais Milliseconde topographie (Nicolas Bernier et Delphine Measroch). II s'agissait d'une installation submersible dont le public pouvait faire l'expérience en s'immergeant littéralement dans la piscine du Centre sportif de l'Université du Québec à Montréal.

Au moment de célébrer son $15^{\mathrm{e}}$ anniversaire, en novembre 2006, Réseaux bouclera la boucle par une soirée consacrée, comme sa toute première, au compositeur Francis Dhomont, dont on célébrera alors le $80^{\mathrm{e}}$ anniversaire. Le bilan qu'affichera l'organisme à ce moment-là nous semble pour le moins méritoire, tant pour son apport indiscutable au développement d'une nouvelle génération d'électroacousticiens que pour la persévérance de ses animateurs à défendre l'existence d'un art qui, plus de 50 ans après sa naissance, reste confiné à la marginalité.

\section{BIBLIOGRAPHIE}

BASQue, Nicole (2000), "Évolution de la musique électroacoustique à Montréal", eContact, vol. 3.3, <http://cec.concordia.ca/ econtact/Histories/EvolutionMontreal.htm>.

BAYLE, François (1993), Musique acousmatique, propositions... positions, Paris, Buchet/Chastel-INA-GRM.

DHOMONT, Francis (1991), "Acousmatique, qu'est-ce à dire?", $<$ http://www.electrocd.com/cat.f/imed_9607.not.html\#imed_960 7-0002>.

DHomont, Francis (1995), "Rappels acousmatiques", eContact, vol. 8.2, <http://cec.concordia.ca/contact/contact82Dhom.html>. Nattiez, Jean-Jacques (éd.) (1993), Circuit : musiques contemporaines, vol. 4, n 1-2, Électroacoustique-Québec : l'essor.

Normandeau, Robert (2001), Situation de l'électroacoustique au Canada, <http://cec.concordia.ca/econtact/Profile/Situation Electroacoustique.htm>.

\section{NOTES}

1. Pour un historique détaillé du développement de l'électroacoustique au Québec, se référer à Nattiez (éd.), 1993; également, la revue électronique de la Communauté électroacoustique canadienne eContact, qui présente de nombreux textes intéressants parmi lesquels Basque 2000.

2. Une première brèche avait été ouverte en 1971 à Montréal par Micheline Coulombe Saint-Marcoux, Otto Joachim et Gilles Tremblay, mais le studio fut fermé en 1974 avant d'être rouvert en 1979 par Yves Daoust.

3. En 1987, avec l'apparition du Digital Audio Tape (DAT), se répand l'expression "musique sur support", encore plus courante aujourd'hui que l'on peut transférer la musique aisément sur $C D$, minidisc ou même disque dur.

4. "[...] vers 1974, pour marquer la différence et éviter toute confusion avec les musiques électroacoustiques de scène ou 
d'instruments transformés (ondes Martenot, guitares électriques, synthétiseurs, systèmes audionumériques en temps réel...), François Bayle [ndlr : en 1993] introduit l'expression musique acousmatique comme spécifique d'une musique d'images qui "se tourne, se développe en studio, se projette en salle, comme le cinéma", en temps différé. "À l'usage, écrit-il, ce terme de prime abord sévère, à la fois critiqué et adopté, se trouve maintenant assoupli par une pratique usuelle parmi les communautés de compositeurs pour désigner quasi naturellement ce qui distingue entre toutes les techniques musicales actuelles, celles-là mêmes qui relèvent proprement du support sonore, d'esthétiques aussi variées soient-elles" (Dhomont, 1995).

5. Mouvances Métaphores, IMED 9107/08, réédité SOus IMED 9607 et IMED 9608 .

6. "Le mot acousmatique revient souvent dans les textes qu'on peut lire ici. Mais que veut-il dire? Ce terme est attribué à Pythagore ( $v \mathrm{I}^{\mathrm{e}}$ siècle avant JC) qui dispensait, dit-on, son enseignement - uniquement oral — dissimulé derrière une tenture afin que ses disciples ne soient pas distraits par sa présence physique et puissent concentrer leur attention sur le seul contenu de son message. Plus près de nous, au début du siècle, on trouve encore dans Le Larousse pour tous en deux volumes : "Acousmate. $n$. $m$. (du grec Akousma, ce qu'on entend). Bruit imaginaire ou dont on ne voit pas les causes, l'auteur." " (Dhomont, 1991)
7. La plupart des compositeurs invités à participer aux Rien à voir $1,2,3,4,5,7,9$ et 12 ont aussi eu la bonne grâce de se prêter à des entrevues devant public juste avant leur concert. Ces entrevues étaient humblement menées par l'auteur de ces lignes.

8. Une qualité sonore plus qu'acceptable, mais nécessitant un dispositif encombrant. D'une manière générale, le compositeur diffuse sa pièce depuis un lecteur de disque compact à peu près standard. Le signal (stéréo) est distribué sur une vingtaine de canaux sur la console de mixage (par exemple, 10 pour le côté gauche, 10 pour le côté droit). Chacun de ces canaux est relié à un amplificateur, lui-même, évidemment, relié à un haut-parleur. 9. Louis Dufort s'est d'ailleurs vu confier une soirée complète lors de Rien à voir ( 7 ).

10. Tel qu'il est décrit par l'organisme, dans la liste des membres du Conseil québécois de la musique.

11. Avant d'être spécifique, la description de l'organisme est, prudemment, générale : Son mandat principal est de diffuser sur la place publique le travail accompli par les artistes et les groupes de production en arts médiatiques. Ses outils de promotion sont les concerts, les bases de données, les livres ou toute autre forme de support qui permet aux œuvres de vivre sur scène et aux écrits d'être diffusés.

12. Ces pièces sont disponibles depuis avril 2002 sur le disque Parr(A)cousmatique, paru, évidemment, chez Empreintes DIGITALes (IMED 0264). 


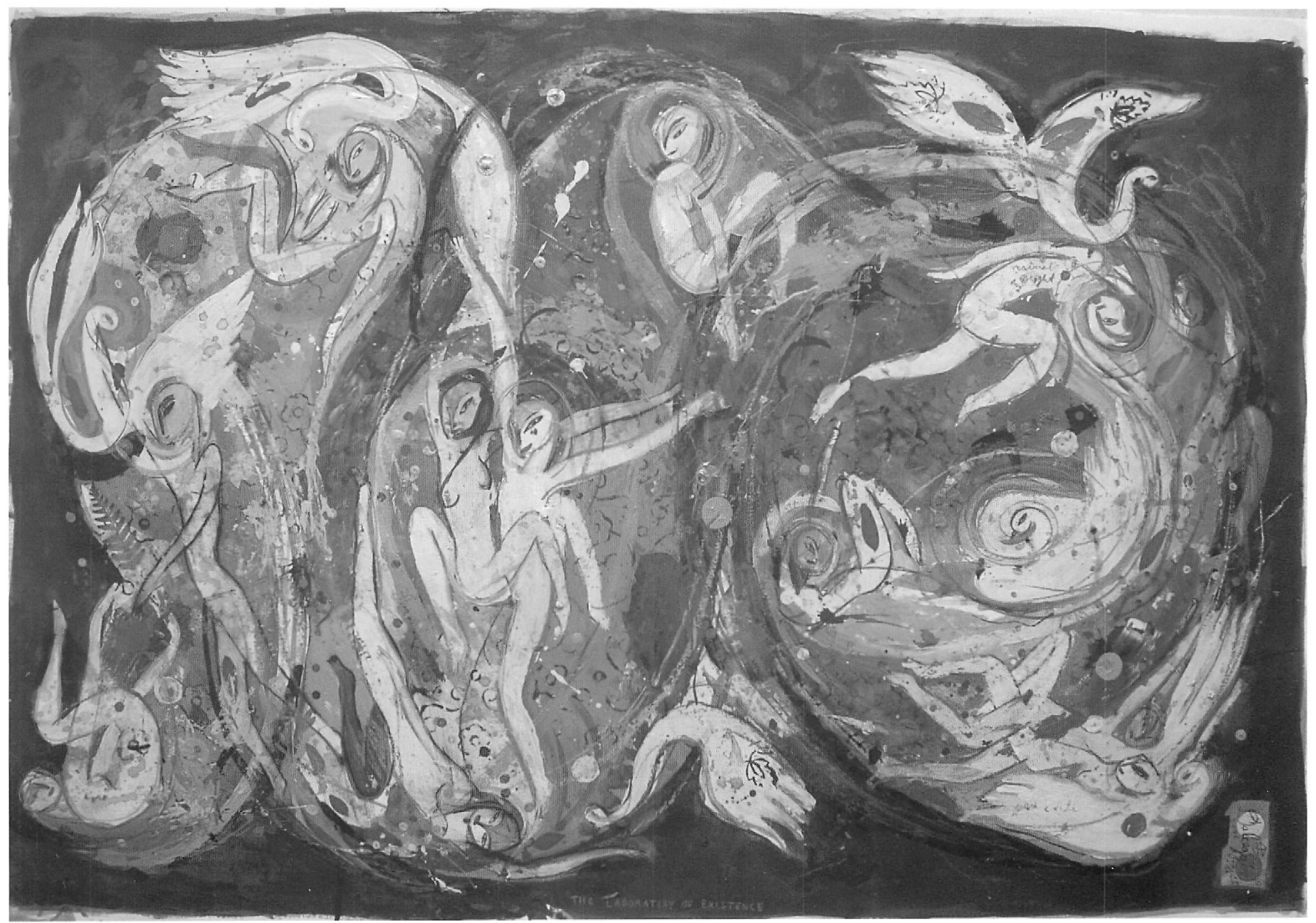

Splendeurs de l'existence (2006, acrylique, encre de Chine, pastel gras sur papier, 30 " X 40") 